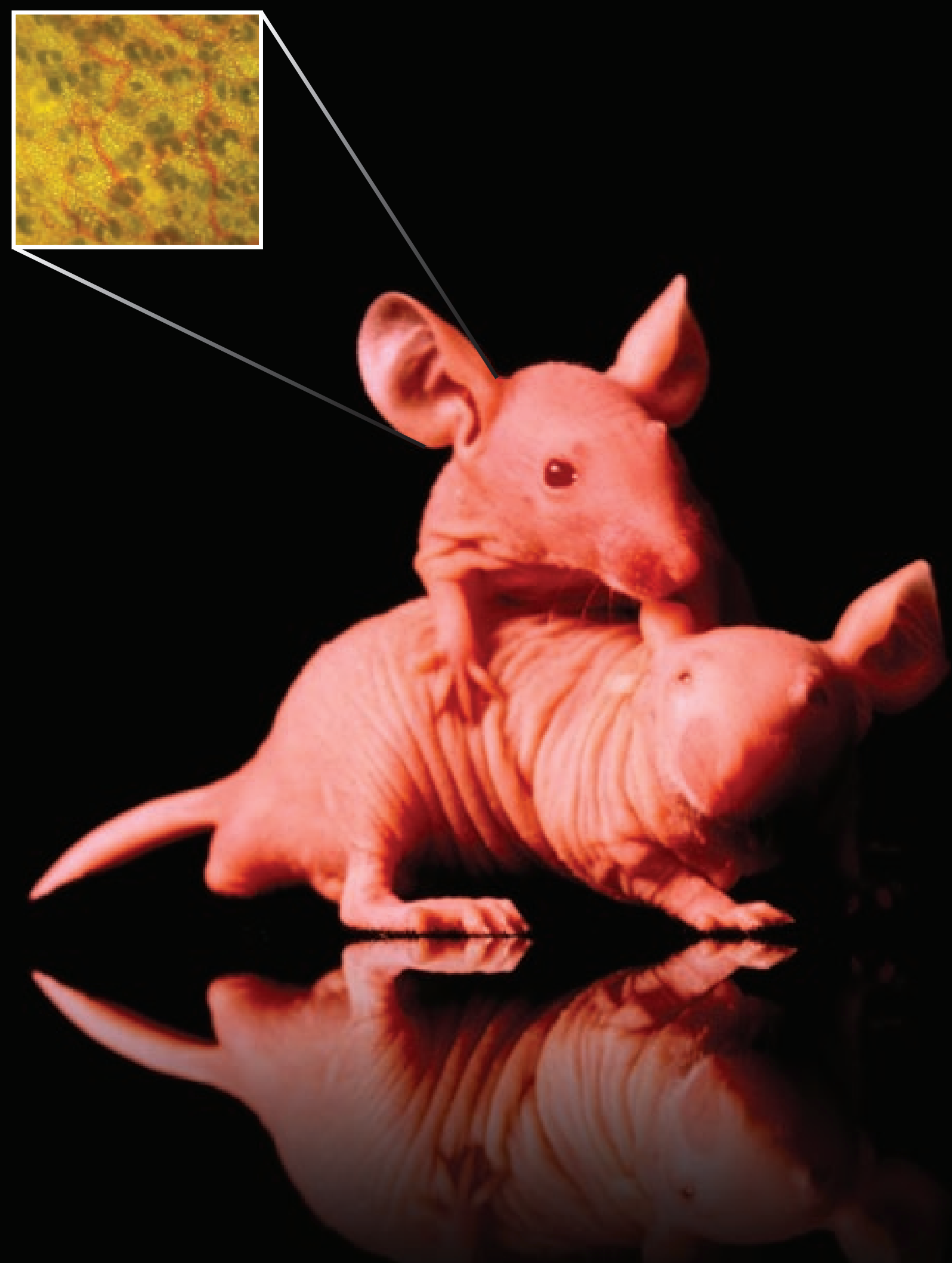

The microvasculature in

the ear of a nude mouse is

captured using transmission

optical microscopy. 


\title{
Photoacoustic Tomography and Microscopy
}

\author{
Lihong V. Wang
}

\section{By converting light waves into sound, researchers have developed a high-resolution biological imaging technique that allows them to see living tissue at new depths.}

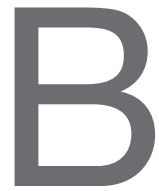

iomedical researchers have greatly benefited from commercially available high-resolution 3D optical imaging modalities_-including confocal microscopy, two-photon microscopy and optical coherence tomography. Unfortunately, however, such tools cannot penetrate biological tissue deeper than about $1 \mathrm{~mm}$. Photoacoustic tomography (PAT), which combines strong optical contrast and high ultrasonic resolution in a single modality, has broken through this fundamental depth limitation.

PAT is cross-sectional or three-dimensional imaging based on the photoacoustic effect, in which light waves are converted to acoustic ones via the absorption of light and its subsequent conversion into heat. Alexander Graham Bell first reported on the photoacoustic effect when he was working on a way to transmit sound without any cables in 1880. It is only recently, however, that scientists have begun to apply photoacoustics to the imaging world.

Optical absorption is the dominant contrast mechanism used in PAT. Intrinsic optical absorption can reveal physiological functions that enable scientists to diagnose cancer and image brain activity, for example. That's because optical absorption changes with the concentration of biological molecules such as oxygenated and deoxygenated hemoglobin and melanin.

By using multi-wavelength measurement, one can simultaneously quantify concentrations of multiple chromophoreswhose presence gives a specific color to a compound-of different colors. (For example, hemoglobin gives its color to red blood cells.) Such quantification of oxygenated and deoxygenated hemoglobin, for instance, can provide functional imaging of the concentration and oxygen saturation of hemoglobin in the blood - which are related to hallmarks of cancer such as hypermetabolism and angiogenesis (the growth of new blood vessels to sustain tumors). Extrinsic optical absorption contrast agents can also be used to image biomarkers.

\section{Challenges in high-resolution optical imaging}

In biological tissues, light transfer is dominated by scattering. The mean free path is on the order of $0.1 \mathrm{~mm}$ and the transport mean free path is roughly $1 \mathrm{~mm}$. While the former measures the frequency of predominantly anisotropic scattering, the latter assesses the frequency of equivalent isotropic scattering. As a result of scattering, photon propagation transitions from the ballistic regime into the diffusive regime at about one transport mean free path.

None of the commercially available optical ballistic imaging modalities-including confocal microscopy, two-photon microscopy and optical coherence tomography — can penetrate beyond one transport mean free path into scattering biological tissue. The reason is that ballistic light attenuates exponentially with a decay constant equal to the mean free path.

By contrast, diffuse optical tomography measures multiplescattered photons and can provide rapid functional imaging beyond this penetration limit. However, it has poor spatial resolution. Until recently, high-resolution optical imaging beyond this penetration limit remained a void. The motivation driving the development of PAT is to combine optical-absorption contrast with ultrasonic spatial resolution at depths greater than the optical transport mean free path. 


\section{General concepts on achieving high resolution}

Suppose we were to try to optically image an embedded structure in the middle plane of a scattering biological object that is much thicker than one transport mean free path. We project a light beam on one side of the tissue and receive the transmitted light on the other. The projection image of the embedded structure is blurred by scattering. Blurring can be reduced by making multiple projections of various view angles followed by reconstructing the image. Even after one takes these steps, however, the best spatial resolution attainable would be approximately 10 percent of the tissue thickness.

If a surgeon excises the right half of the tissue, one can effortlessly acquire a clear image of the embedded structure. The clarity is afforded by the clear propagation of light from the structure to the viewer. The illumination light remains diffusive but does not affect image quality. For the same reason, we can clearly see objects illuminated by broad daylight that is scattered by clouds on a cloudy day.

Less invasively, the right half of the tissue can be made clear using osmotic agents. In postmortem animal studies, optical clearing has drastically reduced scattering. In living tissue, however, the decline in scattering is modest without significant toxicity being induced.

If we can somehow convert the diffuse illumination light on the structure into another form of energy that can propagate clearly through the optically scattering tissue, we can achieve a clear image without cutting or optically clearing the tissue. That's where the photoacoustic effect comes in. Through this effect, light can be transduced into ultrasound, which propagates in tissue with minimum scattering and can therefore provide a clear image of the structure with optical contrast.

\section{Optical contrast and ultrasonic resolution}

In PAT, a researcher irradiates tissue with a short-pulsed laser beam. Absorbed light is then converted into heat, which is further transformed into a rise in pressure via thermoelastic expansion.

The initial increase in pressure-determined by the local optical energy deposition (also known as specific optical absorption) and other thermal and mechanical propertiespropagates as an ultrasonic wave, which is referred to as a photoacoustic wave. The photoacoustic wave can be detected using an ultrasonic transducer, which produces an electric signal. A scientist can then amplify and digitize the signal and transfer it to a computer, where an image is formed.

The image contrast is based on optical absorption in the photoacoustic excitation phase. When a sufficiently short laser pulse illuminates a medium, the local pressure rise immediately following the laser excitation is given by $p_{0}(\mathbf{r})=\beta T(\mathbf{r}) / \kappa$, where $\kappa$ denotes the isothermal compressibility $\left(5 \times 10^{-10} \mathrm{~Pa}^{-1}\right.$ for water or soft tissue), $\beta$ denotes the thermal coefficient of volume expansion $\left(4 \times 10^{-4} \mathrm{~K}^{-1}\right.$ for muscle), and $T$ denotes the temperature rise (K). In soft biological tissue, each $\mathrm{mK}$
[ Imaging through a scattering medium ]

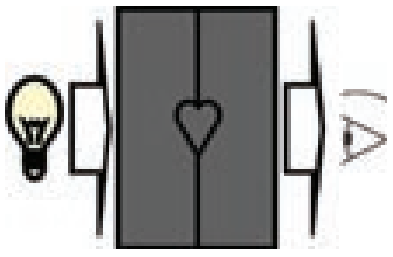

(a)

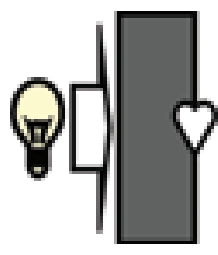

(b)

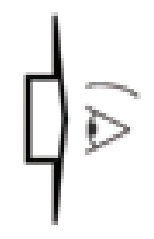

(b)

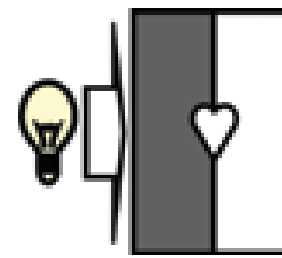

(c)

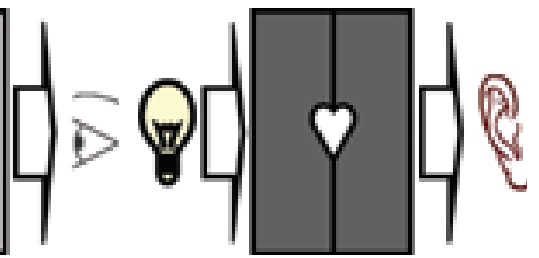

(d) (a) A light beam is projected on one side of a tissue with a structure embedded in the middle plane. The projection image of the embedded structure is blurred by scattering. (b) If the right half of the tissue is surgically removed, a clear image of the embedded structure can be acquired. (c) Using osmotic agents to clear the right half of the tissue also results in a clear image. (d) Through the photoacoustic effect, light can be transduced into ultrasound, which propagates in tissue with minimum scattering and therefore provides a clear image of the structure with optical contrast.
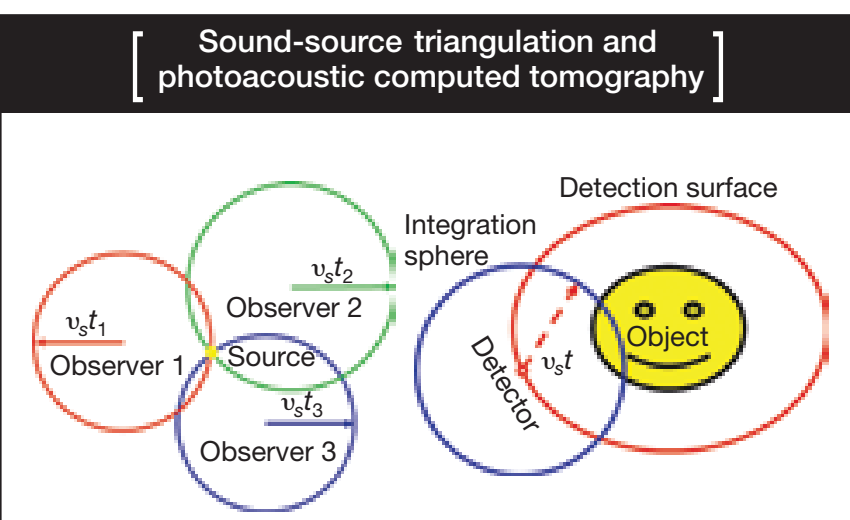

(Left) Simple imaging by triangulation of a single sound source such as thunder. In this schematic, $v_{s}=$ the speed of sound in the medium and $t_{1}, t_{2}$, and $t_{3}=$ propagation times from the sound source to the three observers, respectively. (Right) Detection for photoacoustic computed tomography. Laserinduced photoacoustic signals integrate over a spherical surface centered at each ultrasonic detector placed on the detection surface and can be reconstructed mathematically to form an image of optical contrast. Here, $t=$ time after the laser pulse illumination of the object. 
temperature rise yields an 8-mbar (or $800-\mathrm{Pa}$ ) pressure rise. Because the increase in temperature is related to the optical absorption coefficient of the tissue, imaging $p_{0}$ maps the optical absorption contrast.

The spatial resolution is derived from ultrasonic detection in the photoacoustic emission phase. Ultrasonic scattering is two to three orders of magnitude weaker than optical scattering. As a result, the photoacoustic wave provides better resolution than the optical wave at depths beyond one optical transport mean free path.

\section{Imaging of a sound source by triangulation}

Let us illustrate how to pinpoint a single sound source such as thunder in 3D space, as shown in the bottom figure on $\mathrm{p}$. 38. If the time delay between seeing lightening and hearing thunder is recorded, one can infer that the thunder took place on a spherical surface of a radius given by the product of the speed of sound and the time delay.

The source of the sound could be accurately triangulated as long as one were able to obtain three such measurements at different locations. If the sound source is produced by the photoacoustic effect, photoacoustic tomography can be used to image the source, which is spatially distributed in most cases.

\section{Photoacoustic computed tomography}

Photoacoustic computed tomography is based on measurement with unfocused ultrasonic transducers - ideally point transducers - followed by computer reconstruction. For spherical, cylindrical and planar detection configurations, where the detection surface $S_{0}$ encloses the source $p_{0}(\mathbf{r})$, the analytical back-projection can be expressed by the formula:

$$
p_{0}(\mathbf{r})=\frac{2}{\Omega_{0}} \int_{S_{0}}\left[p\left(\mathbf{r}_{0}, t\right)-t \partial p\left(\mathbf{r}_{0}, t\right) / \partial t\right]_{t=\left|\mathbf{r}-\mathbf{r}_{0}\right| / v_{s}} d \Omega
$$

where solid angle $\Omega_{0}=2 \pi$ for the planar geometry, $\Omega_{0}=4 \pi$ for the spherical or cylindrical geometry, $p\left(\mathbf{r}_{0}, t\right)$ denotes the acoustic pressure measured at position $\mathbf{r}_{0}$ and time $t$, and $d \Omega$ denotes the solid angle subtended by the detector area with respect to the reconstruction point $\mathbf{r}$. Note the time delay between $\mathbf{r}$ and $\mathbf{r}_{0}$.

\section{Photoacoustic microscopy}

Researchers have implemented dark-field confocal photoacoustic microscopy (PAM) using a scanning focused ultrasonic
[ Photoacoustic microscopy system ]

Nd: YAG pump Laser

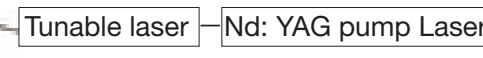

Photodiode

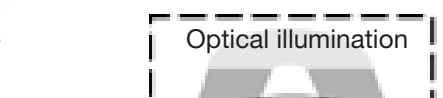

U Ultrasonic ।
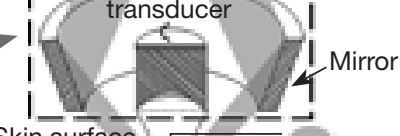

Dual foci

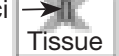

Annular illumination with a dark center transducer. Acoustic coupling requires contact with the biological tissue, as shown in the figure above.

The components within the red dashed-lined box are translated in a water tray, which has a window at the bottom that is sealed with an optically and ultrasonically transparent disposable polyethylene membrane. After a researcher applies a thin layer of ultrasound gel to the region of interest, he or she places the tissue between the water container and the sample support.

Image formation starts from the laser. The researcher uses laser pulses (duration, $6.5 \mathrm{~ns}$ ) from a wavelength-tunable dye laser, which is pumped by an Nd:YAG laser, to produce photoacoustic signals. He or she delivers laser light through an optical fiber to the scanner. For hemoglobin imaging, the laser wavelength is typically set in the Q-band of hemoglobin (560 $\mathrm{nm})$ to achieve a good signal-to-noise ratio.

The researcher records the energy of each laser pulse with a photodiode for shot-to-shot calibration. The laser beam from the fiber passes through a conical lens to form a ring-shaped illumination pattern. It is then weakly focused into the tissue with the focal region coaxially overlapping the ultrasonic focus. The optical illumination on the skin surface is donutshaped with a dark center. Therefore, the photoacoustic signal from the tissue surface in the field of view is minimized.

In an optically clear medium, the optical focus is about $2 \mathrm{~mm}$ in diameter, which is much wider than the ultrasonic focus. The researcher records the photoacoustic wave at each location of the ultrasonic transducer for $2 \mu$ s and subsequently converts it into a 1D depth-resolved image (A-line) based on the sound velocity in soft tissue $(1.54 \mathrm{~mm} / \mu \mathrm{s})$. Then, he or she 
performs raster scanning of the dual optical-ultrasonic foci in the horizontal $(x-y)$ plane with a step size of $50 \mu \mathrm{m}$ to produce a 3D image. No signal averaging is required.

This system is safe for human imaging because it delivers a fluence of only about $6 \mathrm{~mJ} / \mathrm{cm}^{2}$ at the optical focus (pulse energy: $0.2 \mathrm{~mJ}$ ), which is well within the ANSI safety standard $\left(20 \mathrm{~mJ} / \mathrm{cm}^{2}\right)$ in the visible spectral region (400 to $\left.700 \mathrm{~nm}\right)$.

\section{Spatial resolution of photoacoustic microscopy}

Ultrasonic detection with an acoustic lens approximates image reconstruction in photoacoustic computed tomography with a limited detection aperture. While the lateral resolution is determined by the focal diameter of the ultrasonic transducer at the center frequency, the axial resolution is inversely proportional to the bandwidth of the ultrasonic transducer. Therefore, achieving high spatial resolution requires a high center frequency, a large numerical aperture (NA) and a wide bandwidth.

In our $50-\mathrm{MHz}$ PAM system, the ultrasonic detector has a center frequency of $50 \mathrm{MHz}$, an NA of 0.44 , and a nominal bandwidth of $35 \mathrm{MHz}$. To produce photoacoustic signals around this frequency, one should choose a laser with a pulse width less than $1 / 50 \mu$ s or $20 \mathrm{~ns}$. The imaging system has a lateral resolution of roughly $45 \mu \mathrm{m}$ and an axial resolution of $15 \mu \mathrm{m}$. We have reached an imaging depth of more than $3 \mathrm{~mm}$ in biological tissue.

\section{Photoacoustic imaging of vasculature and melanoma}

PAM can be used to image hemoglobin-containing vasculature and melanin-rich melanoma with high intrinsic contrast. At the 584-nm optical wavelength, it can image both melanoma and the surrounding blood vessels in the $x-y$ plane because both melanin and hemoglobin have comparably strong absorption at this wavelength. However, light at this wavelength is unsuitable for measuring the tumor thickness because it cannot easily penetrate the melanin-rich tumor.

Therefore, we formed another image using 764-nm nearinfrared light, which can infiltrate the tumor because of the decreased optical absorption of melanin and hemoglobin. The combination of the two images reveals the 3D morphology of the melanoma and the surrounding vasculature, where some parallel arteries and veins are evident. In the pseudo-colored PAM images shown in the figure below, the blood vessels from the 584-nm image are shown in red while the melanoma from the 764-nm image appears in brown.

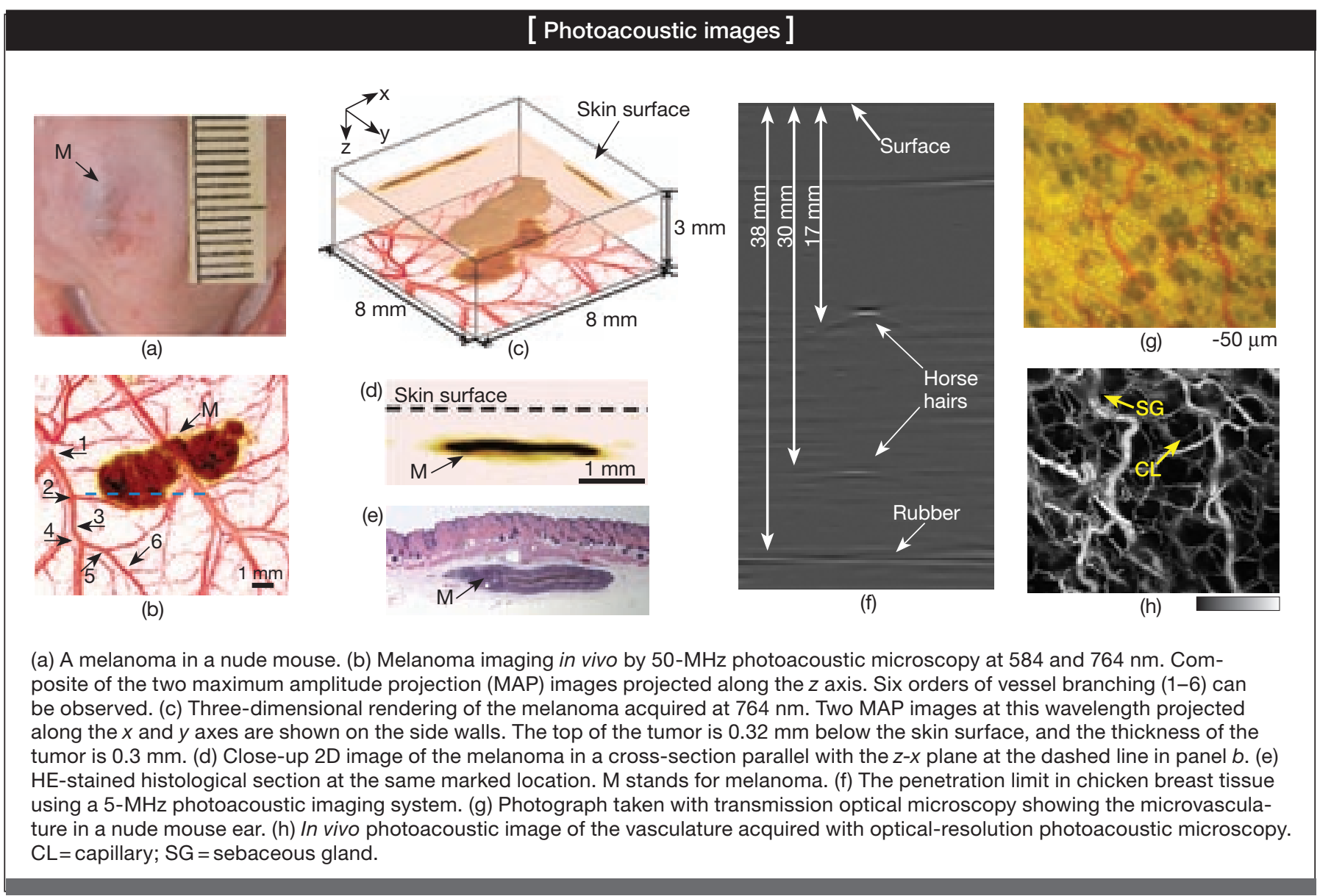




\begin{tabular}{|l|c|c|}
\hline \multicolumn{3}{|c|}{$\left[\begin{array}{c}\text { Comparison of high-resolution } \\
\text { optical imaging modalities }\end{array}\right]$} \\
\hline Modality & Penetration & Primary contrast \\
\hline $\begin{array}{l}\text { Confocal } \\
\text { microscopy }\end{array}$ & $\sim 0.5 \mathrm{~mm}$ & $\begin{array}{l}\text { Scattering, } \\
\text { fluorescence }\end{array}$ \\
\hline $\begin{array}{l}\text { Two-photon } \\
\text { microscopy }\end{array}$ & $\sim 0.5 \mathrm{~mm}$ & Fluorescence \\
\hline $\begin{array}{l}\text { Optical coherence } \\
\text { tomography }\end{array}$ & $\sim 1 \mathrm{~mm}$ & $\begin{array}{l}\text { Scattering, } \\
\text { polarization }\end{array}$ \\
\hline $\begin{array}{l}\text { Confocal photo } \\
\text { acoustic microscopy }\end{array}$ & $\begin{array}{l}\sim 3-30 \mathrm{~mm}, \\
\text { scalable }\end{array}$ & Absorption \\
\hline
\end{tabular}

At $584 \mathrm{~nm}$, the average ratio of the vessel to the background in photoacoustic signal amplitude is $13 \pm 0.9$. At $764 \mathrm{~nm}$, the average ratio of the melanoma tumor to the background is as high as $68 \pm 5$. Such high optical contrast and the associated specificity are due to the minimal background absorption and the strong absorption of both hemoglobin and melanin. Further, PAM provides images devoid of speckle artifacts, which are conspicuous in images acquired with either ultrasonography or optical coherence tomography.

In addition to functional imaging of total hemoglobin concentration, PAM can provide functional imaging of oxygen saturation of hemoglobin using multiwavelength measurements.

\section{Multiscale imaging with common signal origins}

PAM can provide multiscale imaging in vivo with the same contrast origins, which will likely provide an important tool for research on multiscale biology. The image resolution, as well as the depth limit, is scaleable with the ultrasonic frequency within the reach of photons.

Employing a 5-MHz ultrasonic transducer and an 804-nm light source has enabled penetration of $30 \mathrm{~mm}$ in chicken breast tissue. Researchers have also achieved $5 \mu \mathrm{m}$ lateral resolution using a strongly focused light beam, allowing in vivo imaging of capillaries, the smallest blood vessels. Of course, optical focusing is most effective only within the optical transport mean free path.

\section{Comparison of high-resolution optical imaging modalities}

PAM combines high ultrasonic resolution and strong optical contrast in a single modality. It has broken through the fundamental penetration limit at the optical transport mean free path. The various modalities complement each other in both penetration and contrast.

We believe that PAT will find broad applications in both basic research and clinical care. For example, it can potentially be applied in tumor studies, where angiogenesis is highly correlated with cancer aggressiveness; in neuroscience, perhaps with the help of cranial windows, where hemoglobin oxygen saturation and total hemoglobin concentration are related to neural activities; in cancer radiotherapy and chemotherapy, where hypoxia is responsible for resistance to therapy; in trauma evaluation, where optical absorption is associated with both hemorrhage and edema; and in endoscopic imaging, when miniaturized optical and ultrasonic components are integrated into a single probe.

Researchers have identified the following specific clinical applications: melanoma imaging; demarcation for Mohs surgery; treatment planning and monitoring for laser therapy of port-wine stains; skin burn imaging for skin graft; intraoperative demarcation of brain tumors; intra-operative imaging of brain functions; sentinel lymph node mapping; and breast cancer detection. We have no doubt that many more applications will be identified in the future. While the research community continues to push the frontier of PAT, several companies are actively commercializing photoacoustic imaging technologies. We look forward to seeing immense impact of PAT on biomedicine. $\triangle$

This work was sponsored by National Institutes of Health grants R01 EB000712 and R01 NS46214.

[ Lihong V. Wang (LHWang@biomed.wustl.edu) is with the Optical OSA Imaging Laboratory in the department of biomedical engineer$\underline{\text { Member }}$ ing at Washington University in St. Louis, Mo., U.S.A. ]

\section{[ References and Resources ]}

>> A.A. Oraevsky and L.V. Wang. Photons Plus Ultrasound: Imaging and Sensing, SPIE, Vol. 6856 (2008).

>> X.D. Wang et al. "Noninvasive laser-induced photoacoustic tomography for structural and functional in vivo imaging of the brain," Nature Biotech. 21, 803-6 (2003).

>> M.H. Xu and L.V. Wang. "Universal back-projection algorithm for photoacoustic computed tomography," Phys. Rev. E 71, 016706 (2005).

>> M. Xu and L.V. Wang. "Biomedical photoacoustics," Review of Scientific Instruments 96, 163902 (2006).

>> H.F. Zhang et al. "Functional photoacoustic microscopy for highresolution and noninvasive in vivo imaging," Nature Biotech. 24, 848-51 (2006).

>> K. Song and L.V. Wang. "Deep reflection-mode photoacoustic imaging of biological tissue," Journal of Biomedical Optics 12 060503 (2007).

>> L.V. Wang and H. Wu. Biomedical Optics: Principles and Imaging, Wiley, Hoboken, N.J., 2007.

>> H.F. Zhang et al. "In vivo imaging of subcutaneous structures using functional photoacoustic microscopy," Nature Protocols 2 , 797-804 (2007).

>> K. Maslov et al. "Optical-resolution photoacoustic microscopy for in vivo imaging of single capillaries," Opt. Lett. 33, 929-31 (2008).

>> L.V. Wang. "Tutorial on photoacoustic microscopy and computed tomography," IEEE Journal of Selected Topics on Quantum Electronics 14, 171-9 (2008).

>> For taped presentations on photoacoustic tomography and related imaging technologies, please visit http://oilab.seas.wustl.edu. 